\title{
Exploring Crisis Communication and Information Dissemination on Social Media: Social Network Analysis of Hurricane Irma Tweets
}

\author{
Xianlin Jin ${ }^{1}$ (B)
}

1. Ph.D. candidate, College of Communication and Information, University of Kentucky, Lexington, KY, USA

\begin{abstract}
This study utilized social network analysis to identify the top 10 Twitter influentials during the Hurricane Irma crisis period and examined the relationship between social media attributes and the bridge influence of controlling information flow. The number of a user's followers and tweets significantly predicted one's control of information. Crisis information tended to be shared in scattered subgroups. Social network boundaries impeded information diffusion, and the communication pattern was largely one-way. The findings partially supported the opinion leader argument while indicating that influentials can directly generate information, which is consistent with the social-mediated crisis communication model. Such findings will contribute to crisis literature and help emergency management professionals advance social media usage to disseminate crisis information, build effective communication, and provide immediate disaster relief responses.
\end{abstract}

KEYWORDS: crisis influence, opinion leader, social network analysis, social-mediated crisis communication

\section{Introduction}

While facing crises, the public experiences real uncertainties and concerns. To minimize the impact of a crisis and reduce the public's concerns and uncertainties, crisis managers need to provide

CONTACT Xlanlin Jin (D) - Email: xianlin.jin@uky.edu • College of Communication and Information, University of Kentucky, 206 McVey Hall, 155 Graham Ave, Lexington, KY, 40506, USA 
sufficient information and effective communication in a timely manner. Social media platforms, such as Facebook and Twitter, have been shown to facilitate information dissemination, enable ordinary citizens to share ideas and information, and hence allow organizations and members of the public to develop interactive communication during crises (Sadri et al., 2017; Spence et al., 2015). Unfortunately, how crisis communication and information spread on social media is still unclear.

To answer this question, this study explored the bridge influence of controlling information flow and highlighted the importance of analyzing Twitter users' position in the network to identify crisis information influentials. By conducting a social network analysis, this study recognized the top 10 influentials in a crisis and unpacks crisis communication and information dissemination patterns. This study also examined the relationship between social media attributes (e.g., the number of followers, tweets, followed, and favorites) and influence levels.

In particular, this study collected real-time data-tweets surrounding Hurricane Irma. At its strongest, Hurricane Irma was a Category 5 hurricane with winds of $185 \mathrm{mph}$ (National Weather Service, 2017). This crisis caused an estimated 129 deaths (Issa et al., 2018) and a total cost of $\$ 50$ billion in the United States (Cangialosi et al., 2018). It created uncertainties about returning to normal life. With a focus on examining the social-mediated crisis communication and information dissemination patterns of Hurricane Irma, 31,349 tweets and 16,627 Twitter accounts were analyzed. Based on the betweenness centrality (the number of shortest paths that a Twitter user connects other pairs of users, see Hansen et al., 2011), this study discovered the top 10 influential actors who can distribute information and tend to share some features, such as showing media backgrounds, having a large number of followers, and posting many tweets. The reciprocity during the communication process was low and hence the communication pattern was largely one-way.

Moreover, in-degree links were shown to mediate the relationship between the number of a user's followers and the user's central status in the network. In contrast, out-degree links did not mediate the relationship between the number of accounts that a 
user had followed and one's ability in controlling information flow within the network. Furthermore, the number of followers and tweets significantly predicted users' levels of influence within the network.

These findings shed light on the future of theory building and practice for multiple reasons. The findings illustrate the crisis information flow surrounding Hurricane Irma on Twitter and clarify the confusion due to contradictory findings of the relationship between social media attributes and bridge influence. Discovering the characteristics of the top influentials can also help crisis management practitioners track crisis information on social media, provide prompt and accurate information, and eventually reduce the public's uncertainties.

\section{Social-Mediated Communication and Information Flow during Crises}

Members of the public need prompt and accurate information to reduce their uncertainty in order to make appropriate decisions and cope with crises. Social media has shown to not only provide real-time information for the public but also allow people to generate and share information (Morris et al., 2010; Sin \& Kim, 2013). Unfortunately, developing effective social-mediated crisis communication remains a challenge. As shown in prior crisis studies, government organizations were less active compared to ordinary users and should have fully used social media as a primary crisis communication tool (Lachlan et al., 2014; Lin, Spence, et al., 2016). Understanding how information flows on social media would advance crisis theory building and guide crisis management practice. Opinion leaders have been shown to impact information flow in both traditional media and social media (Austin et al., 2012; Dubois \& Gaffney, 2014; Jin \& Liu, 2010; Katz \& Lazarsfeld, 1955; Watts \& Dodds, 2007).

\section{Opinion Leader and the Two-Step Flow of Communication}

Traditionally, research on personal influence focused on the twostep flow of communication that can be traced back to Katz and Lazarsfeld's (1955) research on opinion leaders, defined as "the 
individuals who were likely to influence other persons in their immediate environment" (p. 3). According to the two-step flow of communication theory, a small group of opinion leaders is the information medium between the mass media and the public. In other words, information flows from the mass media through influentials to their followers (Katz \& Lazarsfeld, 1955; Merton, 1968; Watts \& Dodds, 2007). Specifically, the two-step flow of communication theory highlighted four features of being an influential: having followers, being viewed as an expert, being knowledgeable about the topic, and being in a position to exert social pressure or support (Dubois \& Gaffney, 2014).

The argument of opinion leaders developing their influence has been well examined in mass media contexts (Weimann, 1994). However, it remains unspecified how influentials shape opinions and how the mechanism of information dissemination transfers from influentials to their followers or across entire communities (Watts \& Dodds, 2007). This issue turns out to be more complex with the growth of social media that enables the public to voice their opinions and share crisis information as well as experiences with others (Marken, 2007). Different from traditional one-way communication, social media has the possibility to implement interactive communication among emergency management organizations and the public (Spence et al., 2015). The boundary between media professionals and non-elites on social media is not as clear as it is with traditional mass media. The shift to two-way, interactive communication may also impact crisis information flow and social influence diffusion.

\section{Social-Mediated Crisis Communication Model}

Previous research has shown that people used social media during crises to get emotional support, to seek insider information, and to maintain involvement with family and friends (Austin et al., 2012; Lachlan et al., 2014). According to the social-mediated crisis communication (SMCC) model (Jin \& Liu, 2010), influential social media users create crisis information. Social media followers consume such information, and social media inactive members may be indirectly exposed to such information through social media 
followers and/or traditional media impacted by influential social media creators (Austin et al., 2012). This argument suggests that influential social media users can become information providers rather than merely working as an information medium, which contradicts the two-step flow hypothesis. Previous research supports this argument by identifying the unique communication functions of influentials on Twitter, such as influentials sharing information and supporting information exchange during the 2017 Ariana Grande concert bombing (Zhao et al., 2019).

It is necessary to revisit these arguments and examine them with real-time social media data. To unpack the complex patterns of social-mediated crisis communication and information flow, this study explored how influential social media users impact information dissemination and crisis communication surrounding Hurricane Irma.

\section{Influential Users on Social Media}

Communication scholars have devoted tremendous efforts to unpack the social-mediated crisis information dissemination. Many studies in the literature have largely used the frequency of being retweeted as the criteria to evaluate information influence (Lachlan et al., 2014; Lin, Lachlan, \& Spence, 2016; Lovejoy et al., 2012; Rainear et al., 2018; Sutton et al., 2015). For example, some studies focus on developing content analysis with certain samples (Lin, Lachlan, \& Spence, 2016). Many studies examine the relationship between tweet formats (e.g., URL, hashtags, and multimedia files, Lachlan et al., 2014) and the frequency of a tweet being retweeted (Lovejoy et al., 2012; Rainear et al., 2018; Sutton et al., 2015).

Another common approach to determine influential actors was based on the number of their followers (Cha et al., 2010; Wu et al., 2011). This approach assumed that if an actor can be heard by others in the network, the actor is influential (Bakshy et al., 2011; Subbian \& Melville, 2011). With these two approaches, some of the previous studies advocated certain strategies that can be used to promote the visibility of crisis information and increase specific emergency management organizations' influence in crisis communication. 
Nevertheless, the frequency of being retweeted or the number of followers only reflects one aspect of an influential-having followers. Interestingly, as indicated by some research, having millions of followers does not necessarily represent the success of disseminating broadcasted tweets (Cha et al., 2010). These contradictory findings indicate that previous operationalizations did not capture some aspects of the multifaceted concept of opinion leaders. An influential is often seen as an expert by others; the influential shows knowledge and is in a position to exert social pressure (Dubois \& Gaffney, 2014). These aspects have been largely ignored by previous research. These findings also suggest that scholars need to study the information network to identify information, influential users, and track information flow on social media.

To map influentials in crisis communication and information dissemination, this study utilized a social network analysis, a theoretical and methodological approach investigating social relationships and flows between people and other connected entities (Freeman, 2004; Sommerfeldt \& Kent, 2015; Zhao et al., 2018). This author argued that the relationships between an influential actor and other actors are crucial while examining crisis information communication patterns and information flow.

\section{Social Network Analysis}

A social network articulates a social relationship or structure among individuals, social groups (e.g., family, school, company), and connected information communities, such as URLs and Twitter (Bandyopadhyay et al., 2011; Sommerfeldt \& Kent, 2015). Social network analysis has been applied in multiple disciplines, such as management consulting, sociology, and public health (Borgatti et al., 2009). Nevertheless, this approach has not been fully utilized in examining social-mediated crisis communication. As previous research posited, investigating the "web of relationships" advanced the knowledge about the distribution of information and social support through networks (Valente, 2010; Valente \& Rogers, 1995). 


\section{Information Distribution and Social Media Attributes}

Sadri et al. (2017) analyzed Hurricane Sandy tweets and examined how the in-degree, out-degree, eccentricity, and closeness centrality impact information spreading (e.g., tweeting frequency). They found that Twitter users were more active in information distribution if they were less eccentric in the network. Sadri et al. also observed some clusters in which actors were more connected with each other and some isolators in the network. In addition, networks tend to be less transitive while larger subgraphs are more assortative (Sadri et al., 2017). Nevertheless, Sadri et al. did not further identify how a Twitter user's social media attributes impact the user's active role in the network.

Kim (2017) more closely analyzed social media attributes of influential actors by studying the information distribution of the hashtag AlphaGo. According to Kim, information was reciprocal among media sources, the public, and opinion leaders. In particular, opinion leaders were described by Kim as people who served as conversation starters, influentials, active engagers, network builders, and/or information bridges. Kim concluded that the number of accounts that a user has followed significantly predicted the user's central role in the information network. However, Kim's conclusions were built on examining the top 20 influential users' attributes while ignoring other actors' attributes. The significance of these results may be exaggerated. It is necessary to include all users while further exploring how users' attributes impact their influence levels. Moreover, based on Kim's description, conversation starters and influentials showed many in-degree links and affected receivers' opinions. Such vague and overlapping descriptions of conversation starters and influentials indicate unclear operationalizations of influence.

\section{Measuring Influence}

While investigating information flow on social media, many researchers assessed influence with various approaches. For instance, Cha et al. (2010) categorized influentials based on in-degree that presents how popular a Twitter user was in the network. Dubois and Gaffney (2014) further operationalized political influence with 
five dimensions: in-degree, egocentric centrality (extended connections of a Twitter account), clustering coefficient, the ratio of a user's tweets containing keywords to all sampled tweets, and the frequency of being mentioned. According to Dubois and Gaffney, media outlets, journalists, and politicians were identified as influential based on in-degree and eigenvector centrality. However, political commentators and bloggers appear to be influentials based on their interactions with other users and message quality. These different conclusions regarding influential actors stem from the vague interpretation of the concept of influence.

Recent studies on social media influence in crises started mapping users' positions in certain networks to help researchers identify influentials. For instance, Zhao et al. (2018) conceptualized four dimensions of influence on social media: output, reactive outtake, proactive outtake, and network positioning. Specifically, Zhao et al. measured output based on the number of tweets a user posted and the time length of engagement; they assessed proactive outtake by examining the number of retweets, favorites, and followers. They measured reactive outtake by calculating the number of mentions, replies, and positive references. Last, Zhao et al. operationalized network positioning based on the degree centrality, betweenness centrality, as well as PageRank.

The inconsistent conceptual and operational definitions of influence reflect the growing need for social-mediated crisis communication research to be further explored. Meanwhile, these incoherent findings due to the different definitions of influence also create challenges for communication scholars to identify crisis influentials on social media and unpack the complex crisis information flow.

Various existing studies have already explored the features of being an influential, such as having a large number of followers (Cha et al., 2010; Wu et al., 2011) and presenting a long engagement time on social media (e.g., Zhao et al., 2018). The current study did not replicate these efforts. Instead, it examined influence based on the opinion leader concept and concentrated on the core of the opinion leader's role in the two-step flow communication hypothesis-as the intermediary in the information flow (Katz \& Lazarsfeld, 1955; Merton, 1968; Watts \& Dodds, 2007). 
Guided by the argument of opinion leaders (Katz \& Lazarsfeld, 1955), the SMCC model (Jin \& Liu, 2010), and previous research, this study explored Twitter users' control of information to evaluate each actor's influence in the network. In particular, this study focused on bridge influence on Twitter-betweenness centrality that measures the shortest path between two users. When a Twitter user can easily serve as the information "bridge" that connects others, the user is influential in the network. The assumption of betweenness centrality echoes with the two-step flow communication hypothesis that highlights the influential's position of intermediating information flow. The current study embraces Dubois and Gaffney's (2014) idea that an influential user should be in a position to spread information. By examining the betweenness centrality in the crisis information network on Twitter, this study can identify the bridge influentials who are in the central position of the network.

Another goal of this study is to explore the relationship between a user's social media attributes and the user's bridge influence (betweenness centrality). Specifically, the social media attributes include the number of followers a Twitter user has presented, the total number of tweets a user has posted since joining Twitter, all the favorites the user has received, and the number of other accounts that the user has followed. As a side note, although Twitter changed "favorites" to "likes" in 2015 (Whitten, 2015), to be consistent with prior studies and to make a clear comparison with previous findings, the current study used the term of "favorites."

Prior research exploring influence on social media has shown that influential users have more followers (e.g., Cha et al., 2010; Kim, 2017; Wu et al., 2011). However, it is still unclear how the number of followers impacts a Twitter user's influence levels. The current study aims to answer this question. The author argues that when a Twitter user has lots of followers, they are more visible and hence are more likely to show bridge influence in the information network. The visibility argument has been supported by Treem et al. (2020). The number of followers a user has, as indicated by Kim's findings (2017), was also shown to be positively associated with one's in-degree links and betweenness centrality. It should be noted that these findings may be exaggerated because only the 
top influential users' attributes have been analyzed. This paper re-examined the relationships among followers, in-degree links, and betweenness centrality by analyzing the attributes and influence levels of all Twitter users in the information network surrounding Hurricane Irma. Therefore, inspired by previous findings, opinion leaders research (Dubois \& Gaffney, 2014; Katz \& Lazarsfeld, 1955), and the SMCC model (Jin \& Liu, 2010), this paper posited that when one Twitter user is being followed by many accounts, the user is likely to be seen as an information provider reflected by the number of in-degree links (edges pointing toward the user) and hence is in a central position in the network.

H1: The more followers that a user has, the more in-degree links that the user would receive, resulting in the user being more central in the information network.

Similarly, if one Twitter user follows many accounts, the user will see many information resources and hence will be able to seek information from multiple sources. In other words, the user will present more out-degree links (edges leaving the user). As such, this study hypothesized that this user will likely be in a central position in the network.

H2: The more accounts that a user has followed, the more outdegree links that the user would present, resulting in the user being more central in the information network.

Besides being in the central position in a network, an influential is also characterized as being knowledgeable and therefore can generate information about a specific topic (e.g., the total number of tweets). To capture this feature of influence, this study also explored how the total number of tweets a user has posted predicts betweenness centrality.

Moreover, an influential is seen as an expert (Dubois \& Gaffney, 2014; Katz \& Lazarsfeld, 1955) and thereby an influential actor's posts should be valued. The degree to which a user's posts are valued can be assessed by the number of favorites that the user received. The author argued that these social media attributes predict Twitter users' influential levels. Following the logic, this paper 
also investigated relationships between users' social media attributes (followers, following, tweets, and favorites) and the bridge influence.

$\mathrm{H}_{3}$ : The number of accounts that a user followed, the number of followers, the number of tweets that a user posted, as well as the favorites that a user has received will predict the levels of influence in the network.

The ultimate purpose of this study is to improve the assessment of crisis information flow and crisis communication patterns. By developing a social network analysis, this paper explored the information network's density and reciprocity. The following research questions were proposed and examined.

RQ1: Who are the influential actors in the network of Hurricane Irma information dissemination?

RQ2: Through what patterns does information flow in the network?

\section{Method}

\section{Data Collection}

To include relevant information about Hurricane Irma on Twitter, the author checked the crisis period. Hurricane Irma formed on August 30, 2017, and turned into an extratropical storm after September 12 (National Oceanic and Atmospheric Administration, 2017; Weather Underground, 2017). The author collected 31,349 tweets (original tweets and retweets posted from September 1 to September 14, 2017) and 16,627 Twitter accounts with NodeXL Pro (Smith et al., 2010) that connects to the public Application Programming Interface of Twitter. Specifically, the author searched the keywords "Hurricane Irma" and "HurricaneIrma" to collect data. The keywords searching strategy is advantageous because this approach enables the author to collect all relevant data rather than limiting data to tweets with specific hashtags. The current dataset involved tweets with different hashtags, such as \#HurricaneIrma, \#Irma, \#Florida, and \#US. 


\section{Data Analyses}

This study conducted a series of social network analyses with NodeXL Pro (Smith et al., 2010). In particular, to answer RQ1 and RQ2, each user's betweenness centrality, in-degree links, and out-degree links were calculated. In addition, with the ClausetNewman-Moore clustering algorithm, subgraphs were conducted to identify the crisis network subgroups about Hurricane Irma. The clustering coefficient, reciprocated edge ratio, network density, and average geodesic distance were calculated as well. Table 1 describes the explanation of each metric.

\section{TABLE 1 Metric Description}

\begin{tabular}{|l|l|}
\hline Metric & Description \\
\hline In-degree Links & $\begin{array}{l}\text { The number of directed edges pointing toward a } \\
\text { specific node. In this study, it refers to the number } \\
\text { of Twitter accounts that seek information from a } \\
\text { certain Twitter user. }\end{array}$ \\
\hline Out-degree Links & $\begin{array}{l}\text { The number of outgoing connections leaving } \\
\text { from a specific node. In this study, it represents the } \\
\text { number of links that a specific Twitter account used } \\
\text { to seek out information. }\end{array}$ \\
\hline $\begin{array}{l}\text { Betweenness } \\
\text { Centrality }\end{array}$ & $\begin{array}{l}\text { The extent to which a Twitter user works as an } \\
\text { information "bridge" along the shortest path } \\
\text { between other users. }\end{array}$ \\
\hline $\begin{array}{l}\text { Clustering } \\
\text { Coefficient }\end{array}$ & $\begin{array}{l}\text { The degree to which a Twitter user is embedded } \\
\text { within a graph where all users tend to tightly cluster } \\
\text { together. }\end{array}$ \\
\hline Network Density & $\begin{array}{l}\text { The ratio of edges relative to the number of all } \\
\text { possible edges. It reflects the cohesiveness in the } \\
\text { network. }\end{array}$ \\
\hline $\begin{array}{l}\text { Reciprocated } \\
\text { Edge Ratio }\end{array}$ & $\begin{array}{l}\text { The portion of edges that have reciprocated } \\
\text { interactions. In this study, it reveals the information } \\
\text { exchange between Twitter users and reciprocity of } \\
\text { communication patterns. }\end{array}$ \\
\hline Geodesic Distance & $\begin{array}{l}\text { The length of the shortest geodesic between two } \\
\text { Twitter users. }\end{array}$ \\
\hline
\end{tabular}


To test $\mathrm{H}_{1}$ and $\mathrm{H}_{2}$, mediation tests were conducted with the Model 4 of Process Macro Version 3 (Hayes, 2018). Standardized $z$ scores of each variable were calculated and then used in the mediation tests. This study also examined how a Twitter user's attributes impact information dissemination. To test $\mathrm{H}_{3}$, a regression test was conducted with SPSS. Particularly, considering that the tweet dataset is not normally distributed and contains zero, cube root scores of each variable were calculated prior to conducting the regression test. The following attributes were the predictors examined in the linear regression: the number of other accounts that a Twitter user has followed, the sum of followers that a user has, the total number of tweets that a user has posted, and favorites that a user has received.

\section{Results}

The average reciprocated ratio of the crisis information network surrounding Hurricane Irma was .02. The average geodesic distance was 5.44 and the network density (graph density) was $7.47358 \mathrm{E}-$ 05 . The top 10 influential users in the information network were identified based on betweenness centrality (See Table 2). The largest betweenness was 25,551,947.96. Among the top 10 influential actors, two were official agency accounts and five were users who demonstrated media backgrounds. United States President Donald Trump's Twitter account was the fourth most influential user in terms of his central status in the network (betweenness centrality is 12,944,744.83). Compared to other influential users, President Trump's account showed the most in-degree links (865). However, it had zero out-degree links in the current study's sample.

Regarding RQ2, this study identified 773 subgroups. The top three subgroups that contained the most edges were identified. The largest subgroup contained 4,773 edges including the fifth most influential user in the network. The second-largest subgroup had 3,684 edges including the official Twitter account of the Federal Emergency Management Agency (FEMA). The third-largest subgroup involved 3,078 edges, such as the Twitter accounts of President Trump, Fox News, and New York News. It is worth mentioning that the official Twitter account of the National Weather Service Miami-South Florida was in a group with only two edges. 
TABLE 2 Top 10 Influential Actors in the Hurricane Irma Information Network (September 1 to September 14, 2017)

\begin{tabular}{|c|c|c|c|c|}
\hline User ID & Profile Description & $\begin{array}{c}\text { In- } \\
\text { Degree }\end{array}$ & $\begin{array}{l}\text { Out- } \\
\text { Degree }\end{array}$ & Betweenness \\
\hline 4williamlewis & $\begin{array}{l}\text { Radio host, social } \\
\text { media whiz, political } \\
\text { consultant }\end{array}$ & 0 & 261 & $25,551,947.96$ \\
\hline 4billlewis & $\begin{array}{l}\text { Former AM talk } \\
\text { show host }\end{array}$ & 2 & 247 & $22,293,112.15$ \\
\hline Rebschmdt & $\begin{array}{l}\text { Political freelancer, } \\
\text { previously an online } \\
\text { editor }\end{array}$ & 745 & 1 & $15,265,799.82$ \\
\hline Potus & President Trump & 858 & 0 & $12,944,744.83$ \\
\hline Hsiglobal & $\begin{array}{l}\text { Humane Society } \\
\text { International }\end{array}$ & 90 & 1 & $11,667,847.63$ \\
\hline Miketheiss & $\begin{array}{l}\text { National Geographic } \\
\text { Photographer \& } \\
\text { Storm Chaser }\end{array}$ & 469 & 3 & $11,513,647.44$ \\
\hline 2jenniferlewis & Been suspended & 0 & 76 & $10,332,601.08$ \\
\hline 4rickstaly & $\begin{array}{l}\text { A graduate of } \\
\text { the FBI National } \\
\text { Academy, law } \\
\text { enforcement }\end{array}$ & 0 & 74 & $10,168,626.99$ \\
\hline marishkamistry & $\begin{array}{l}\text { WWF Member } \\
\text { \& Animal Rights } \\
\text { Campaigner }\end{array}$ & 0 & 2 & $9,301,017.14$ \\
\hline Duvalschools & $\begin{array}{l}\text { Duval County Public } \\
\text { Schools }\end{array}$ & 342 & 1 & $8,051,535.68$ \\
\hline
\end{tabular}

This study discovered the communication patterns of subgroups by conducting subgraphs in NodeXL Pro (Smith et al., 2010). Figure 1 shows the position of FEMA's Twitter account in the second-largest subgroup. Specifically, FEMA's account had 167 in-degree links, two out-degree links, and its betweenness centrality was $4,883,617.58$ with a clustering coefficient of 0.004 . At the time of data collection, FEMA had followed 640 Twitter accounts with 675,144 followers; it had posted 12,884 tweets and received 
FIGURE 1 FEMA's Status in the 2nd Largest Subgroup/Subgraph.

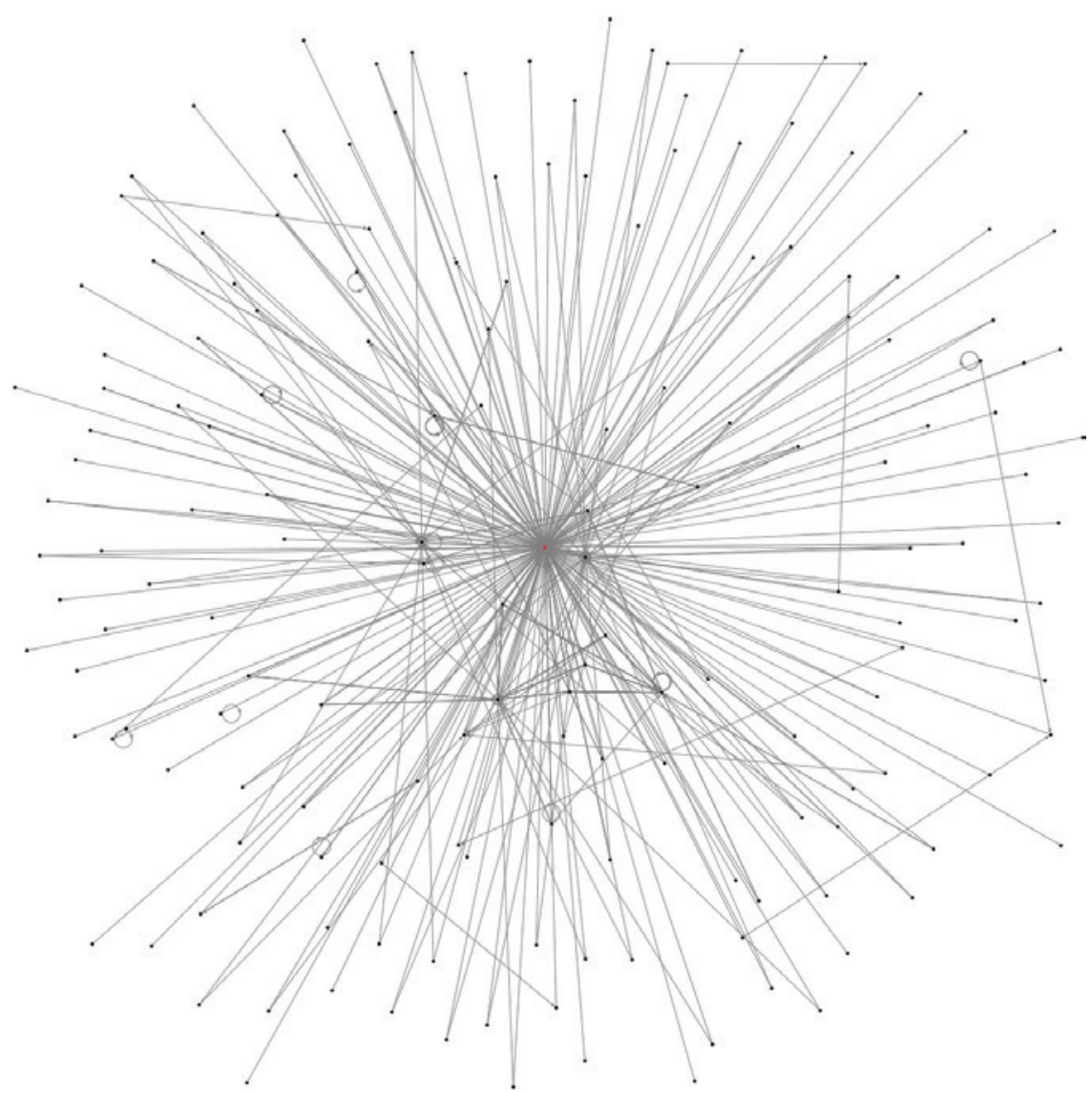

2,534 favorites. Figure 2 presented the status of President Trump's Twitter account in the third-largest subgroup. President Trump's account had 858 in-degree links, zero out-degree links, and the betweenness centrality was $12,944,744.83$ with a clustering coefficient of 0.002 . At the time the dataset was collected, President Trump's account had followed 42 other accounts and had been followed by 20,263,131 accounts. He had posted 1,169 tweets and received 84 favorites. 
FIGURE 2 President Trump's Status in the 3rd Largest Subgroup/ Subgraph.

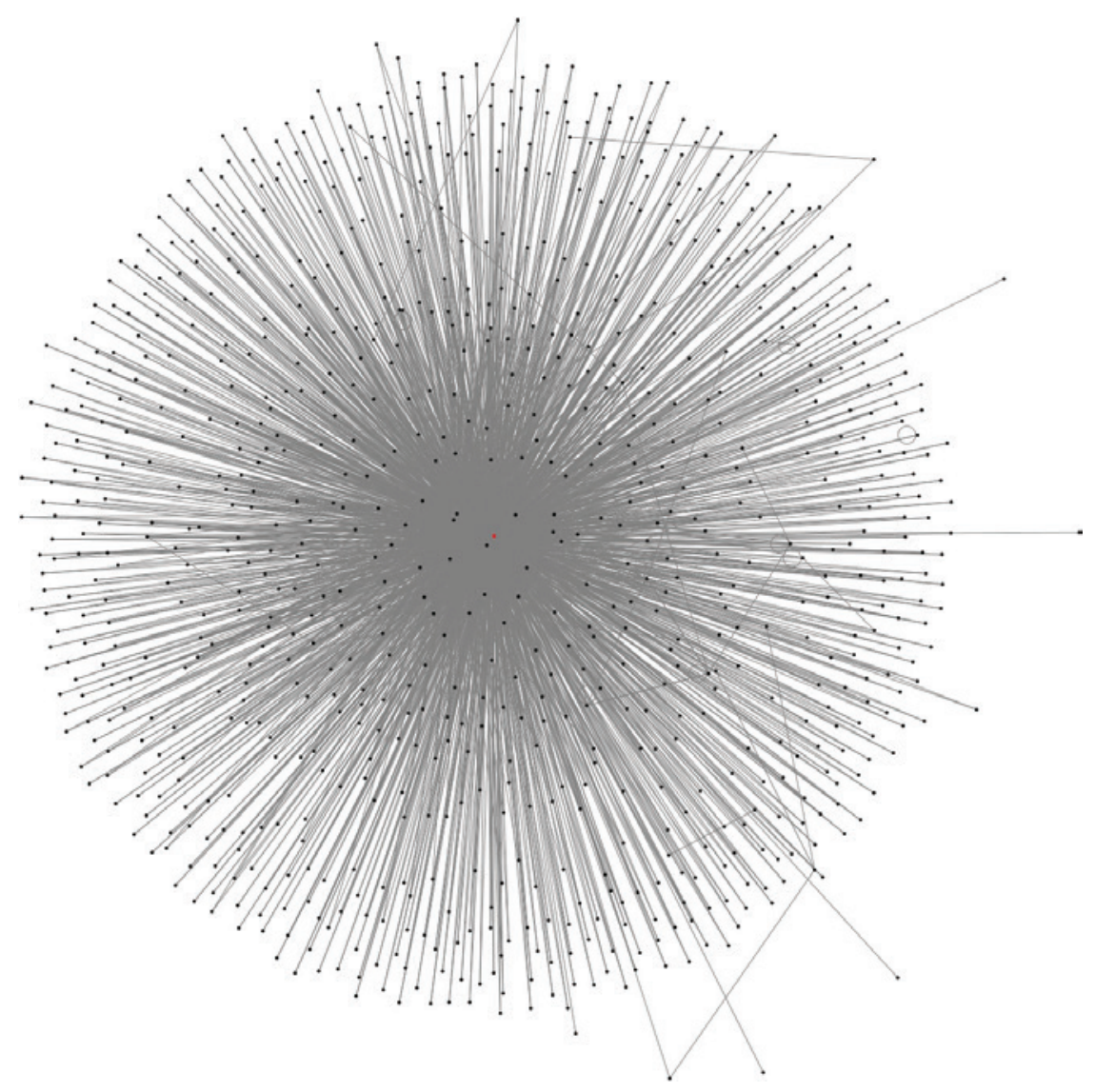

The correlations between all variables were provided in Table 3 . The correlation between the number of tweets and favorites presented the largest effect size (.58). $\mathrm{H}_{1}$ was supported. The number of followers a user has showed a significant indirect impact on the user's betweenness centrality through in-degree links, $\mathrm{b}=$ $.17,95 \%$ CI $[.1462, .1996]$ (Figure 3 ). In addition, the regression relationship between followers and in-degree links was positive and statistically significant $(\beta=.40, t(16625)=19.61, \mathrm{SE} .=.02$, $p<.05)$. Both in-degree links $(\beta=.42, t(16624)=20.21, \mathrm{SE}=.02$, $p<.05)$ and the number of followers $(\beta=.08, t(16624)=5.23$, $\mathrm{SE}=.01, p<.05)$ significantly predicted a Twitter user's betweenness centrality. 


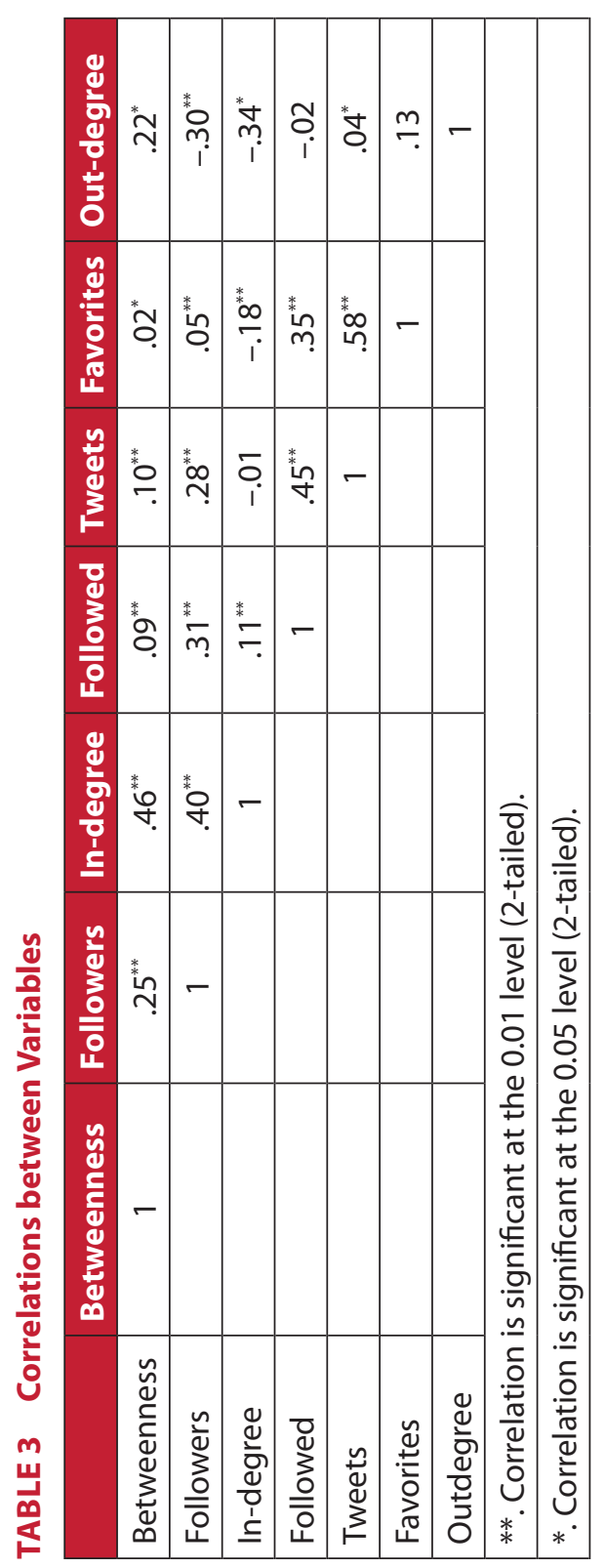


FIGURE 3 Standardized Regression Coefficients for the Relationship between the Number of Followers and the Betweenness Centrality as Mediated by In-degree Links.

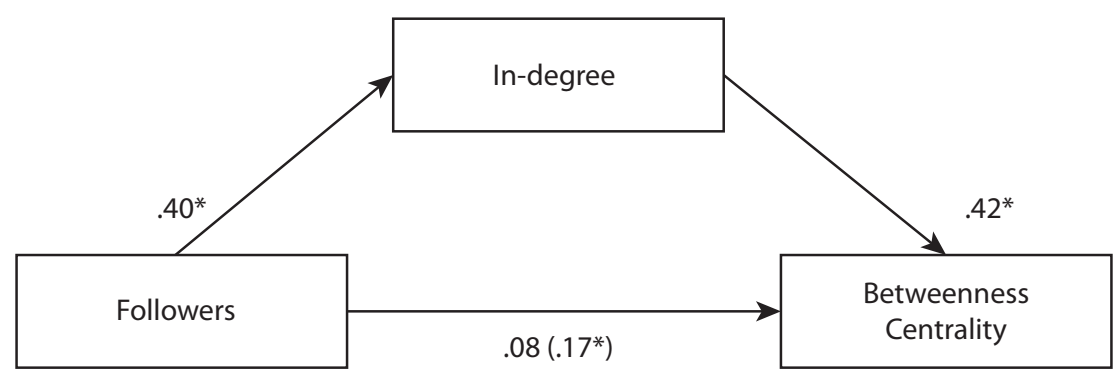

Note: The indirect effect coefficient is in parentheses. ${ }^{*} p<.05$.

$\mathrm{H}_{2}$ was not supported. There was no statistically significant mediation impact of out-degree on the number of accounts a user followed and the betweenness centrality: $b=.00,95 \% \mathrm{CI}[-.0053$, $.0005]$. Moreover, no statistically significant linear relationship was found between the number of accounts that a user has followed and the number of out-degree links that a user has shown: $\beta=.00, t(16625)=-.50, \mathrm{SE}=.01, p=.62$. Interestingly, both out-degree links $(\beta=.36, t(16624)=49.45, \mathrm{SE}=.01, p<.05)$ and the number of accounts that a user has followed $(\beta=.02, t(16624)$ $=3.30, \mathrm{SE}=.01, p<.05)$ were found to show statistical significance of predicting betweenness centrality.

$\mathrm{H}_{3}$ was partially supported. The number of followers $(\beta=.23$, $t(16622)=28.64, p<.05)$ and tweets $(\beta=.04, t(16622)=4.32$, $p<.05)$ both showed positive and statistically significant relationship with betweenness centrality in the information network of Hurricane Irma. In particular, the number of followers showed a medium effect size $(\beta=.23)$. The number of favorites that the user has received was negatively related to the user's betweenness centrality in the information network $(\beta=-.02, t(16622)=-2.31$, $p<.05)$. However, the number of other accounts that a user has followed did not show statistical significance in predicting betweenness centrality (see Table 4 ). 


\section{TABLE 4 Regression of Attributes and Betweenness Centrality}

\begin{tabular}{|l|c|c|c|} 
& $\boldsymbol{\beta}$ & $\boldsymbol{t}$ & $\boldsymbol{p}$ \\
\hline Constant & & 6.75 & .00 \\
\hline Favorites & -.02 & -2.31 & .02 \\
\hline Followed & .01 & .92 & .36 \\
\hline Followers & .23 & 28.64 & .00 \\
\hline Tweets & .04 & 4.32 & .00 \\
\hline
\end{tabular}

\section{Discussion}

By conducting a social network analysis with tweets about Hurricane Irma that were posted during the crisis period, this study explores patterns of crisis communication and information flow. Inspired by the opinion leader perspective and the two-step flow of communication theory (Katz \& Lazarsfeld, 1955), as well as the SMCC model (Jin \& Liu, 2010), the study examines the multiple facets of information influentials and investigates how influentials impact information flow. It identifies the top 10 influential actors based on their betweenness centrality and analyzes the impacts of a user's social attributes (e.g., followers, following, tweets, favorites) on the user's bridge influence. The findings suggest that the crisis information network remains dispersed and information is largely shared within smaller subgroups.

Numerous studies have examined how message formats impact the frequency of being retweeted (e.g., Lachlan et al., 2014; Lovejoy et al., 2012). However, the broad picture of crisis information flow remains unclear. This study enriches the crisis communication literature by (1) further exploring the bridge influence during the crisis information dissemination on social media, (2) identifying the top 10 influentials in a crisis information network, (3) examining the relationship between social media attributes and bridge influence levels (betweenness centrality), and (4) unpacking crisis information and dissemination patterns. 


\section{Bridge Influence, Information Flow, and Crisis Communication}

This study returns attention to the original definition of influence proposed in the argument of opinion leaders and the two-step flow hypothesis (Katz \& Lazarsfeld, 1955; Merton, 1968; Watts \& Dodds, 2007). The existing social-mediated crisis communication research tends to inconsistently conceptualize and operationalize influence across studies. For example, some studies assess influence based on the number of followers (Cha et al., 2010; Wu et al., 2011), while ignoring these actors' influence over controlling information flow. Facing this challenge, this study intrinsically concentrates on the bridge influence that is evaluated based on a Twitter user's ability to connect others with the shortest path in the network: betweenness centrality.

One unique contribution of this study is to use social network analysis to assess influence, which adds a new approach to opinion leaders and information flow research. Previous research has utilized various ways to measure influence, such as the number of followers, frequency of being retweeted, and in-degree links (e.g., Cha et al., 2010; Lovejoy et al., 2012; Wu et al., 2011). These measures indeed reflect partial aspects of influence. Nevertheless, the key features of influence are understudied, that is, the influencer's capacity to generate information as the SMCC model (Jin \& Liu, 2010) hypothesized and/or to intermediate information flow as the opinion leaders argument posited (Katz \& Lazarsfeld, 1955; Merton, 1968; Watts \& Dodds, 2007). This study pays attention to the control of information dissemination and evaluates Twitter users' influence levels based on betweenness centrality. This study argues that betweenness centrality reflects a user's role as an information bridge during the information dissemination process.

\section{Characteristics of Influentials}

As opinion leaders in a crisis information network, influential Twitter users showed the following characteristics: demonstrating media backgrounds, having a large number of followers, having posted many tweets, and presenting more connections with other users (e.g., attracting more links pointing inward from others and/ 
or generating more links to reach other users). In this study's sample, influential Twitter users present the ability to be information resources and directly control information flow.

This study identifies the top 10 influential users in the information network of Hurricane Irma. The finding suggests that ordinary citizens with media backgrounds are active on social media. These social media users have the ability to distribute information online. Federal official accounts, such as the National Weather Service, are not as active as some individual accounts, which echoed previous studies (Lachlan et al., 2014; Lin, Spence, et al., 2016). Non-elites can be opinion leaders who connect their community with information. Being media outlets or journalists is not the premise of being opinion leaders. Presenting media skills, instead, helps ordinary users become opinion leaders. As shown in this study's sample, many top influential Twitter accounts rarely seek out information (fewer out-degree links), such as President Trump. However, they are often seen as an information provider. This finding suggests that social media influentials can directly generate and spread information as the SMCC model (Jin \& Liu, 2010) proposed, a contrast to Katz and Lazarsfeld's (1955) argument that highlights opinion leaders' role of intermediating the flow between mass media and the public. Indeed, this finding needs to be further examined in the future because this study only analyzes visible information interactions and does not examine the content of information.

\section{Social Media Attributes and Bridge Influence}

This study also further explains the relationship between a user's social media attributes and the user's bridge influence. During the process, the study analyzes the multifaceted characteristics of influence, such as having followers, being knowledgeable, being seen as experts, and being in a central position to exert influence (see Dubois \& Gaffney, 2014). The study's findings emphasize the importance of analyzing opinion leaders' positions within a network to capture the patterns of information flow.

This study identifies how a Twitter user's social media attributes predict the user's bridge influence-the extent to which the 
user can connect to others with the shortest path (betweenness centrality). According to the mediation analysis results, the number of followers directly impacts bridge influence. It also indirectly predicts bridge influence through in-degree links that are the number of links pointing inward and reflect the degree to which others come to the specific user for information. Both the number of followers and in-degree links predict a user's central status in the information network about Hurricane Irma. Contrary to previous research in which a large number of followers was not related to successful information dissemination (Cha et al., 2010), this study finds that when a Twitter user has more followers, the user is more likely to be seen as an information provider (reflected by showing more in-degree links). The correlation between followers and in-degree links is positive and the effect size is moderate. Compared to the number of followers, a Twitter user's ability to control information flow is more correlated to in-degree links. The complex findings may suggest that the impact of followers on a user's influence levels may not be oversimplified by the number of followers; instead, the user's chances of being reached by others (in-degree links) play a more important role in shaping the user as an information bridge. If only a few followers came to a user for information, the user would not exert a considerable influence on the information flow.

In this study's data, even though both out-degree links and the number of following (accounts that a user has followed) impact the user's ability to serve as an information bridge, out-degree links do not mediate the relationship between the number of following and the betweenness centrality. Moreover, compared to the number of following, out-degree links explained more variances in the user's control of information flow. The correlation between the number of following and the betweenness centrality is weak. The complex results indicate that a user's ability to seek information from other users essentially predicts the user's central position in the network.

Consistent with the previous research (Kim, 2017), this study also shows that the number of following is not associated with out-degree links. This finding suggests that even if a user has followed many accounts on Twitter, the user does not necessarily 
reach out to look for crisis information (out-degree links). Instead, previous studies indicate many people tend to use social media for emotional support and involvement (Austin et al., 2012; Lachlan et al., 2014). Some Twitter users may not treat other accounts as the primary and credible resource to seek crisis information. Another possible interpretation is that even if a Twitter user has followed many accounts, this user does not necessarily read accessible posts. Only if a user actively seeks information on Twitter, will the user spread information. In addition, Twitter algorithms may impact the individual user's access to specific information, which requires further examination in the future.

To delve into the relationship between Twitter accounts' social media attributes and their influence levels, this study includes all Twitter accounts in the analysis rather than only analyzing the top 10 influentials' characteristics. This study examines how a user's influence in disseminating information is impacted by the number of the user's followers, following, tweets, and favorites. The latter two factors have been largely ignored by previous studies (e.g., Kim, 2017; Sadri et al., 2017).

In this study's sample, the number of followers and the total number of tweets a user posted positively impact the user's bridge influence in the network. In particular, the number of followers presents the strongest prediction among social media attributes. Even though the number of favorites that the user has received is shown to negatively affect a user's betweenness centrality, this small effect requires further examination in the future. The findings suggest that when one Twitter user has more followers and posts more tweets, the user will exert a stronger influence in distributing information. This study suggests that being knowledgeable and having followers are two important features of being an influential, which partially supports the opinion leader argument (Dubois \& Gaffney, 2014; Katz \& Lazarsfeld, 1955). Previous studies have contradictory findings regarding whether the number of followers shapes a user as an influential (e.g., Cha et al., 2010; Kim, 2017; Sadri et al., 2017; Zhao et al., 2018). The current study also clarifies the relationship between the number of followers and being influential in a crisis information network. 


\section{The Crisis Communication and Information Network Surrounding Hurricane Irma}

This study aims to unpack crisis complexity by conducting a social network analysis with real-time data. The complexity of crises brings more challenges for crisis managers and public relations practitioners. The common approaches using limited samples (e.g., content analysis of tweets, exploring the linear relationship between tweets' format and retweets) may have difficulty fully understanding the chaotic systems of crises. Responding to the challenge, this study collects large and real-time data with NodeXL (Smith et al., 2010).

This study suggests that crisis information is largely shared within some subgroups. The network of crisis communication surrounding Hurricane Irma remains dispersed. The information exchange among Twitter users at a broad level remains insufficient and the communication pattern is mainly one-way. This is consistent with Sadri et al. (2017) while it is contrary to Kim's (2017) findings. The possibility of spreading information across the network remains limited by subgroup boundaries. People still have difficulty with breaking such a boundary. This finding may also suggest that, to some extent, an individual's selection of an online network may match one's offline network in reality. Hence, individuals still cannot break the network border online as they experience it in real-life. Scholars can further examine such network boundaries of information dissemination in the future.

The current study's insights provide implications for theory building in crisis communication, opinion leader, and information flow research. The knowledge helps crisis management practitioners achieve the following goals: promptly monitoring information flow on social media, building effective crisis communication, disseminating accurate information, and reducing the public's uncertainties. Identifying the communication and information flow on social media will assist crisis management institutions (e.g., National Weather Services, FEMA, and state governments) to be aware of the public's concerns and correct any misinformation in a timely manner. Understanding the characteristics of the information network and influentials can help crisis management institutions improve their accounts' visibility 
and better connect to target audiences. Relevant organizations, officials, and concerned individuals can also learn how to build bridge influence to disseminate accurate information, which helps mitigate the public's uncertainty.

\section{Limitations}

As a pilot study exploring crisis communication patterns and information dissemination, this paper has several limitations. This study cannot explore the potential impacts of mass media behind the scenes. For instance, some influential Twitter users may be exposed to and impacted by mass media, and hence they may not be an actual "information source."

Moreover, this study only explores communication patterns and information dissemination on one social media platform during the crisis period. It does not examine the information flow across different crisis stages and on various media platforms. Like previous research, this study also merely examines the number of information sources rather than the quality and credibility of information sources. Researchers can further examine how the quality of information posted by influential actors impact their ability to control the information flow.

Finally, this study only includes three edge types: tweets, mentions (retweets are categorized into mentions), and replies. It does not examine the edge type of "follows" due to the limitation of data collection. Having the last edge type may provide insights regarding why some subgroups are formed and why some actors are isolated in the network. Future research would be needed to compensate for these limitations.

\section{Future Directions}

This study provides some useful insights for future research. First, as discussed earlier, in this study, non-elites show the ability to spread information and impact information dissemination. Many social media influentials can directly guide information flow. Social media allows ordinary users to be information providers. Traditional mass media is no longer the only choice for information seekers. Further research is needed to examine the two-step 
flow of communication hypothesis in a social media environment. This study also suspects that the quality and credibility of information sources may impact individuals' crisis informationseeking on social media. More empirical studies in this field will be needed.

Moreover, previous research largely focused on social media usage during pre-crisis and crisis periods (Lachlan et al., 2014; Spence et al., 2015). It will be helpful to examine how information disseminates across pre-crisis, crisis, and post-crisis and how such communication patterns and information flow of each crisis change across different periods.

Last, whereas crisis communication and public relation studies emphasize the importance of synchronizing crisis information across channels, few theories explain and guide organizations to strategically coordinate communication across traditional media, social media, and interpersonal channels. This gap becomes more important given that social media is increasingly being used along with traditional public relations tools. Future research would be needed to examine information flow across traditional media, various social media platforms, and interpersonal communication channels.

\section{Conclusion}

For theoretical development, this study sheds light on information flow, the argument of opinion leaders, and social-mediated crisis communication research. It offers multiple insights for future research and practice. The current study unpacks the crisis information and dissemination patterns by conducting a social network analysis. It explores the Twitter users' bridge influence to identify the crisis information flow on social media. Moreover, it further examines how a Twitter user's social media attributes (followers, following, tweets, and favorites) impact the user's position in the network and the ability to control information dissemination. This study identifies the top 10 influential actors by calculating betweenness centrality. The knowledge provides implications for researchers to further examine the argument of opinion leaders 
(Katz \& Lazarsfeld, 1955) in a social media context and refine the theory development in crisis influence.

This study finds that demonstrating media backgrounds, showing more connections with other users, and having a large number of followers and tweets will predict one Twitter user's ability to control information flow. The current study's findings also indicate that crisis information is shared within subgroups and crisis communication remains dispersed. These patterns suggest crisis communication and information exchange are limited by the online network boundary. Such knowledge will also help guide emergency management practitioners, government officials, and other authorities to share accurate information and provide immediate responses on social media.

\section{Acknowledgments}

I would like to thank Drs. H. Dan O'Hair, Joseph Ferrare, and Andrew Pilny for their feedback.

\section{ORCID}

Xianlin Jin 이 https://orcid.org/oooo-0002-7691-2984

\section{References}

Austin, L., Liu, B. F., \& Jin, Y. (2012). How audiences seek out crisis information: Exploring the social-mediated crisis communication model. Journal of Applied Communication Research, 40(2), 188-207. https://doi.org/10.1080/00909882.2012.654498

Bakshy, E., Hofman, J. M., Mason, W. A., \& Watts, D. J. (2011). Everyone's an influencer: Quantifying influence on Twitter categories and subject descriptors. Proceedings of the Fourth ACM International Conference on Web Search and Data Mining (pp. 65-74). https://doi.org/10.1145/1935826.1935845

Bandyopadhyay, S., Rao, A. R., \& Sinha, B. K. (2011). One introduction to social network analysis. In S. Bandyopadhyay, A. R. Rao, \& B. K. Sinha (Eds.), Models for social networks with statistical applications (pp. 1-29). Sage Publications, Inc. 
Borgatti, S. P., Mehra, A., Brass, D. J., \& Labianca, G. (2009). Network analysis in the social sciences. Science, 323(5916), 892-895. https://doi.org/10.1126/science.1165821

Cangialosi, J. P., Latto, A. S., \& Berg, R. (2018, June 30). Hurricane Irma (AL112017) tropical cyclone report. National Hurricane Center. Archived at https://web.archive.org/ web/20180613160921/https://www.nhc.noaa.gov/data/tcr/ AL112017_Irma.pdf

Cha, M., Haddadi, H., Benevenuto, F., \& Gummadi, K. P. (2010). Measuring user influence on twitter: The million follower fallacy. ICWSM, 10, 10-17.

Dubois, E., \& Gaffney, D. F. (2014). The multiple facets of influence: Identifying political influentials and opinion leaders on Twitter. American Behavioral Scientist, 58(10), 1260-1277. https://doi.org/10.1177/0002764214527088

Freeman, L. C. (2004). The development of social network analysis: A study in the sociology of science. Empirical Press.

Hansen, D. L., Schneiderman, B., \& Smith, M. (2011). Analyzing social media networks with NodeXL: Insights from a connected world. Morgan Kauffman.

Hayes, A. F. (2018). Introduction to mediation, moderation, and conditional process analysis: A regression-based approach (2nd ed.). The Guilford Press.

Issa, A., Ramadugu, K., Mulay, P., Hamilton, J., Siegel, V., Harrison, C., Campbell, C. M., Blackmore, C., Bayleyegn, T., \& Boehmer, T. (2018). Deaths related to Hurricane Irma-Florida, Georgia, and North Carolina, September 4-October 10, 2017. Morbidity and Mortality Weekly Report, 67(30), 829-832. https://doi. org/10.15585/mmwr.mm6730a5

Jin, Y., \& Liu, B. F. (2010). The blog-mediated crisis communication model: Recommendations for responding to influential external blogs. Journal of Public Relations Research, 22 (4), 429455. https://doi.org/10.1080/10627261003801420

Katz, E., \& Lazarsfeld, P. F. (1955). Personal influence: The part played by people in the flow of mass communications. The Free Press. 
Kim, J. (2017). How did the information flow in the \#AlphaGo hashtag network? A social network analysis of the largescale information network on Twitter. Cyberpsychology, Behavior, and Social Networking, 2o(12), 746-752. https://doi. org/10.1089/cyber.2016.0572

Lachlan, K. A., Spence, P. R., Lin, X., \& Del Greco, M. (2014). Screaming into the wind: Examining the volume and content of tweets associated with Hurricane Sandy. Communication Studies, 65(5), 500-518. https://doi.org/10.1080/10510974.20 14.956941

Lin, X., Lachlan, K. A., \& Spence, P. R. (2016). Exploring extreme events on social media: A comparison of user reposting/ retweeting behaviors on Twitter and Weibo. Computers in Human Behavior, 65, 576-581. https://doi.org/10.1016/j. chb.2016.04.032

Lin, X., Spence, P. R., Sellnow, T. L., \& Lachlan, K. A. (2016). Crisis communication, learning and responding: Best practices in social media. Computers in Human Behavior, 65, 601-605. https://doi.org/10.1016/j.chb.2016.05.080

Lovejoy, K., Waters, R. D., \& Saxton, G. D. (2012). Engaging stakeholders through Twitter: How nonprofit organizations are getting more out of 140 characters or less. Public Relations Review, 38, 313-318. https://doi.org/10.1016/j.pubrev.2012.01.005

Marken, G. A. (2007). Social media...The hunted can become the hunter. Public Relations Quarterly, 52(4), 9-12. https://doi. org/10.1037/e380852004-038

Merton, R. K. (1968). Patterns of influence: Local and cosmopolitan influentials. In R. K. Merton (Ed.), Social theory and social structure (pp. 441-447). Free Press.

Morris, M. R., Teevan, J., \& Panovich, K. (2010). A comparison of information seeking using search engines and social media. Proceedings of the 4th International AAAI Conference on Weblogs and Social Media (pp. 291-294). Association for the Advancement of Artificial Intelligence.

National Oceanic and Atmospheric Administration. (2017). [WPC surface analysis archive]. http://www.wpc.ncep.noaa. gov/archives/web_pages/sfc/sfc_archive_maps.php?arcdate $=09 / 16 / 2017 \&$ selmap $=2017091615 \&$ maptype $=$ satsfcnps 
National Weather Service. (2017). Central Alabama impacts from Irma September 10-12, 2017. http://www.weather.gov/bmx/ event_irma2017

Rainear, A. M., Lachlan, K., Odeldorf-Hirsch, A., \& DeVoss, C. L. (2018). Examining Twitter content of state emergency management during Hurricane Joaquin. Communication Research Reports, 35(4), 325-334. https://doi.org/10.1080/08824096.20 18.1503945

Sadri, A. M., Hasan, S., Ukkusuri, S. V., \& Cebrián, M. (2017). Understanding information spreading in social media during Hurricane Sandy: User activity and network properties. CoRR, abs/1706.03019. https://arxiv.org/ftp/arxiv/ papers/1706/1706.03019.pdf

Sin, S.-C. J., \& Kim, K.-S. (2013). International students' everyday life information seeking: The informational value of social networking sites. Library \& Information Science Research, 35(2), 107-116. https://doi.org/10.1016/j.lisr.2012.11.006

Smith, M., Ceni A., Milic-Frayling, N., Shneiderman, B., Mendes Rodrigues, E., Leskovec, J., Dunne, C. (2010). NodeXL: A free and open network overview, discovery and exploration add-in for Excel 2007/2010/2013/2016. https://www.smrfoundation. org

Sommerfeldt, E. J., \& Kent, M. L. (2015). Civil society, networks, and relationship management: Beyond the organization-Public dyad. International Journal of Strategic Communication, 9(3), 235-252. https://doi.org/10.1080/1553118x.2015.1025405

Spence, P. R., Lachlan, K. A., Lin, X., \& Del Greco, M. (2015). Variability in Twitter content across the stages of a natural disaster: Implications for crisis communication. Communication Quarterly, 63(2), 171-186. https://doi.org/10.1080/01463373. 2015.1012219

Subbian, K., \& Melville, P. (2011). Supervised rank aggregation for predicting influencers in Twitter. Proceedings of the 2011 IEEE Third International Conference on Privacy, Security, Risk, and Trust (PASSAT) (pp. 661-665). https://doi.org/10.1109/passat/ socialcom.2011.167 
Sutton, J., Gibson, B. S., Spiro, E. S., League, C., Fitzhugh, S., Johnson, B., \& Butts, C. T. (2015). What it takes to get passed on: Message content, style, and structure as predictors of retransmission in the Boston Marathon bombing response. PLoS ONE, 10(8), e0134452. https://doi.org/10.1371/journal. pone.0134452

Treem, J. W., Leonardi, P. M., van den Hooff, B. (2020). Computermediated communication in the age of communication visibility. Journal of Computer-Mediated Communication, 25(1), 44-59. https://doi.org/10.1093/jcmc/zmzo24

Valente, T. W. (2010). Social networks and health: Models, methods, and applications. Oxford University Press.

Valente, T. W., \& Rogers, E.M. (1995). The origins and development of the diffusion of innovations paradigm as an example of scientific growth. Science Communication, 16(3), 242-273. https://doi.org/10.1177/1075547095016003002

Watts, D. J., \& Dodds, P. S. (2007). Influentials, network, and public opinion formation. Journal of Consumer Research, 34(4), 441-458. https://doi.org/10.1086/518527

Weather Underground. (2017). Hurricane Irma. Archived at https://web.archive.org/web/20170915153327/https://www. wunderground.com/hurricane/atlantic/2017/hurricaneirma?map=history

Weimann, G. (1994). The influentials: People who influence people. State University of New York Press.

Whitten, S. (November 3, 2015). Twitter swaps 'favorites' for 'likes' and some users aren't happy. Consumer News and Business Channel. Archived at https://web.archive.org/ web/20161030074410/https://www.cnbc.com/2015/11/03/ twitter-swaps-favorites-for-likes-and-some-users-arenthappy.html

Wu, S., Hofman, J. M., Mason, W. A., \& Watts, D. J. (2011). Who says what to whom on Twitter. Proceedings of the 2oth International Conference on World Wide Web (pp. 705-714). https://doi.org/10.1145/1963405.1963504 
Zhao, X., Zhan, M. M., \& Liu, B. F. (2018). Disentangling social media influence in crises: Testing a four-factor model of social media influence with large data. Public Relations Review, 44, 549-561. https://doi.org/10.1016/j.pubrev.2018.08.002

Zhao, X., Zhan, M. M., \& Liu, B. F. (2019). Understanding motivated publics during disasters: Examining message functions, frames, and styles of social media influentials and followers. Journal of Contingencies and Crisis Management, 27, 387-399. https://doi.org/10.1111/1468-5973.12279 